\title{
Situación socioeducativa de la infancia residente en centros penitenciarios de Colombia
}

\section{Socio-educational situation of children living in Colombian penitentiary centers}

\author{
Viviana Ahumada Carriazo \\ Francisco José Del Pozo Serrano \\ Universidad del Norte, Barranquilla, Colombia
}

\section{Resumen}

Objetivo: estudiar la situación socioeducativa de la infancia residente en centros penitenciarios de Colombia. Método: estudio realizado bajo el paradigma interpretativo, con un enfoque cualitativo y método hermenéutico. Se utilizaron entrevistas a cinco profesionales colaboradores dentro de los centros para la recolección de datos, con una muestra de cinco dentro de un universo de ocho centros penitenciarios existentes en total en Colombia con Madres e hijos (Es decir un profesional por cada centro penitenciario). Resultados: se encontró una situación variada pero con condiciones relativamente adecuadas para la atención a primera infancia y sus madres. Discusión y Conclusiones: se plantea la posibilidad de entornos socio-comunitarios que permitan realmente propender por el correcto desarrollo psicosocial de estos niños/as como lo dicta la ley. Para esto, debe ofrecerse un contexto de convivencia con las mismas condiciones y posibilidades de socialización externas.

Palabras clave: Infancia, centros penitenciarios, madres reclusas.

\begin{abstract}
Objective: This paper aims to study the socio-educational situation of children living in different Colombian penitentiaries. Method: this study is based on an interpretative paradigm with a qualitative approach and hermeneutical method. Interviews are used as technique for data collection. Five volunteer professionals and also mothers and children of these Colombian penitentiaries centers were interviewed (that means one professional per center). Results: A varied situation with relatively proper conditions for childcare and their mothers are found in this study. Discussion y Conclusion: However, as part of both conclusions and discussions, sociocommunity environments which allow to provide a correct psico-social development of these children, as it is dictated by state law. A safe environment with same conditions and possibilities of external socialization must be offered, in order to achieve this psico-social development.
\end{abstract}

Keywords: good teaching, memorable teachers, mentors, physical education faculty.
Open Access:

ISSN: $0124-2121$ E-ISSN: $2665-2420$

ARTÍCULO RSULTADO DE INVESTIGACIÓN

Copyright $\odot 2019$

By Educación y Humanismo

Editor:

Patricia Martínez Barrios Universidad Simón Bolivar

\footnotetext{
Correspondencia:

Viviana Ahumada

vcarriazo@uninorte.edu.co
}

Recibido: 2019-04-30 Aceptado: 01-05-19 Publicado: 27-08-19

10.17081/eduhum 21

37.3117 


\section{Introducción}

La población de mujeres en centros penitenciarios ha ido en aumento (Almeda, Di Nella \& Navarro, 2012, Almeda \& Di Nella, 2017). Las cifras colombianas evidencian que del total de la población carcelaria según el Informe estadístico número 7 del INPEC (Instituto Nacional Penitenciario y Carcelario), el 6,6\% corresponde a mujeres (INPEC, 2017a). En esta población se hace presente la aparición de situaciones inherentes al ciclo evolutivo, como lo son la fertilidad y la maternidad, lo cual conlleva a plantearse necesidades específicas en cuanto a salud, educación pero especialmente reeducación.

Si bien los centros penitenciarios han existido desde tiempos antiguos, su manejo, especialmente en épocas pasadas, ha sobresalido por el hecho de castigar más que por reeducar. De hecho "Muchos han puesto en evidencia la gran contradicción que supone querer reeducar a la persona con los negativos condicionantes de la reclusión" (Ayuso, 2000, p.75).

Estos espacios fueron creados inicialmente para hombres. Hoy en día también residen mujeres, sin embargo, las adaptaciones necesarias para recibir a este tipo de población no han sido suficientes y en muchos casos se presentan situaciones discriminatorias (Parlamento Europeo, 2008; Fondo de Desarrollo de las Naciones Unidas para la MujerUNIFEM, 2007), pero adicionalmente existe un tercer tipo de población para la cual es sumamente importante realizar adaptaciones específicas de nivel estructural y metodológica: la población de mujeres con hijos/as. (Añaños, 2012, Del Pozo \& Idárraga, 2015), especialmente para niños y niñas cuyos derechos prevalecen sobre los derechos de los demás (Constitución política de Colombia, 1991, art. 44). Para el 2017, en Colombia existían 11 centros de reclusión de mujeres, 8 de los cuales tienen infantes para sumar un total de 66 niños residentes en centros penitenciarios (INPEC, 2017b).

Para los Estados del mundo, la primera infancia ha ido tomando más importancia en los últimos años. En Colombia, un ejemplo de esto es la apertura de la estrategia De cero a Siempre que busca, entre otras cosas, garantizar los cinco estructurantes planteados: El cuidado y la crianza; la salud, la alimentación y la nutrición; la educación inicial; la recreación; y el ejercicio de la ciudadanía y la participación (Gobierno de Colombia, 2013), que son aspectos trascedentes en la infancia, la cual es definida como un estado y condición de la vida. (Fondo de las Naciones Unidas para la Infancia-UNICEF, 2005). Al respecto, cabe mencionar la importancia del desarrollo infantil que se construye a partir de los retos y los desafíos que tanto niñas como niños, encuentran en sus diferentes contextos teniendo en cuenta la educación como promotora de estos desafíos y papel de la familia en afianzarlos (Jaramillo, 2007, Vila, 2000).

Hablar de infancia, desarrollo y centros de reclusión, nos ubica en temáticas sumamente 
amplias, por ello, cabe focalizar el estudio en los menores residentes en centros penitenciarios, que, por el contexto, son individuos en situación de vulnerabilidad. Surge entonces el interés de indagar sobre aspectos socioeducativos en este contexto, un tema que ha sido poco abordado y del cual es poca la documentación registrada actualmente (Del Pozo, 2017a), especialmente en países como Colombia.

De allí nace la inquietud por estudiar el problema sobre la vulnerabilidad que se puede presentar en el desarrollo de la infancia residente en entornos aparentemente limitados como son los centros penitenciarios y carcelarios de Colombia, esto por medio del estudio de la situación socioeducativa vinculada a la primera infancia residente en estos centros (planteado como objetivo general del proyecto de investigación) y partiendo de este, realizar y dar a conocer una caracterización de esta población con sus madres, analizar el programa de atención integral y proponer medidas socioeducativas de mejora.

Es importante tener en cuenta que para la legislación Colombiana, los/as niños/as menores de tres años pueden permanecer con sus progenitoras en los centros penitenciarios y carcelarios (Congreso de Colombia, 2014, Ley 1709), para muchos sonará descabellado teniendo en cuenta que las madres recluidas no cuentan con insumos para brindarle a los infantes unas condiciones de crecimiento totalmente normales, debido a que se encuentran privadas de su libertad y en espacios que históricamente no han procurado condiciones para un adulto y mucho menos para el crecimiento de un infante. Sin embargo, la tendencia internacional va por esa vía. Estudios e investigaciones, han demostrado que durante la gestación, tanto él bebe como la madre se preparan para su separación física y posterior proceso de desarrollo conjunto; de ahí la importancia de que el/la niño/a crezca al lado de su madre genética los primeros años de vida, pues los beneficios de una atención y crecimiento conjunto son muchos (López, 2009, Morillo \& Montero, 2010). Además, es importante resaltar que, la separación temprana a raíz de la reclusión de la madre, puede causar efectos negativos en el desarrollo del menor (Cortázar, Fernández, Léniz, Quesille, Villalobos \& Vielma, 2015). Y a nivel mental, el vínculo desarrollado en los primeros años de vida con la madre repercute en su salud posterior (Moneta, 2014), por estas razones, surge la necesidad de incluir y favorecer en los centros penitenciarios, espacios para la atención integral de los menores nacidos y residentes, en los cuales se pueda propiciar un desarrollo adecuado (Borja, Jiménez, Narváez \& Polo, 2017).

En esta misma línea, la Declaración Universal de Derechos del Hombre de 1948 y el Pacto Internacional de Derechos Civiles y Políticos de 1966, resaltan a la familia como elemento fundamental con derechos de protección por parte del Estado y la Sociedad; igualmente, el convenio europeo de Roma para la protección de Derechos Humanos y de las Libertades Fundamentales, expone que todas las personas poseen derecho a una vida familiar, y la Cátedra Europea sobre los derechos de los niños/as, declara y reconoce en su artículo 8.17 que los menores tienen derecho a sus padres, a gozar de ellos y en su artículo 8.11 se expone que los Estados deben asegurar a los padres la asistencia oportuna a sus responsabilidades (Naredo, 2007). De esta forma, prevalece la familia y no la separación. 
Así pues, haciendo una relación costo-beneficio, resalta como opción mayormente positiva el hecho de mantener a los niños y niñas con sus madres durante sus primeros años, teniendo en cuenta la importancia del desarrollo de la infancia desde el apego positivo con la madre. Diversos estudios sobre la situación de estos niños, han concluido que su nivel de desarrollo es normal y comparable al de los niños y niñas que crecen en contextos normalizados (Jaffé, Pons \& Rey, 1997, Jiménez, 2008, Jiménez, 2012, López, 2016, Poehlmann, 2005).

Permanece la duda sobre las condiciones en las que crecerán estos infantes, frente a lo cual, la respuesta está encaminada a realizar adaptaciones tanto a niños como a madres, teniendo en cuenta el interés superior del niño/a y las repercusiones negativas que puede traer la fracturación de la relación: madre e hijo.

Analizar el programa de atención a primera infancia y las medidas que se ofrecen desde los centros penitenciarios enfocados en la atención integral, permite crear un panorama de las condiciones de encierro y las repercusiones que pueden tener en el desarrollo de los/as infantes, quienes, al ser seres sociales, deben tener un contacto permanente con su entorno para un óptimo desarrollo social e integral (Campo, 2014, Torres, 2018).

Es importante tener en cuenta aspectos como los actores contemplados en los procesos educativos. Vila (2000) afirma que "La educación infantil tiene tres actores: niños y niñas, familias y profesionales de la educación" (p.41). Colocando como responsables principales a profesionales de la educación y familias, y concibiendo a niños y niñas como centro de la misma, sin desconocer que la sociedad en general es también corresponsable y benefactora si se tiene en cuenta la función educativa de la escuela y la familia en la comunidad (Alcocer, 2011, Mir, Batle \& Hernández, 2009).

Es importante hacer alusión a la educación social cómo mediadora del problema dentro de los procesos institucionales, ligada entre otras cosas al concepto de protección social e igualdad (Marcó, 2014, Cecchini, Filgueira \& Robles, 2014, Rico \& Trucco, 2013). Del Pozo (2012) afirma que la educación social tiene potencial en acciones educativas dirigidas a "colectivos, personas o situaciones especialmente excluidos, multi-problemáticos o conflictivos. Por ejemplo, se puede plantear la educación social en temas que la literatura, las instituciones y la ciudadanía colombiana ha resaltado como de atención urgente: La salud penitenciaria" (p. 78). Es así como, por medio de la educación social, se pueden encontrar vías de mejoramiento para espacios penitenciarios, estableciendo adaptaciones para la población de madres con niños y niñas, lo cual brinda una salida a los procesos institucionales. 


\section{Metodología}

Esta investigación se llevó a cabo entre los años 2017 y 2018 con insumos investigativos de diferentes partes de Colombia que cuentan con centros penitenciarios en donde residen mujeres y niños recluidos. El paradigma que enmarca esta investigación es interpretativo, propio de las ciencias sociales que concibe el concepto de realidad como algo construido, hace énfasis sobre lo cualitativo, es abierto, emergente inductivo y holístico (González, 2001, Sánchez, 2013).

La investigación es de tipo cualitativo, teniendo en cuenta que es inductiva, ya que se encuentra relacionada con el descubrimiento y el hallazgo que con la comprobación o la verificación, es holística, no se reduce al estudio de variables sino a un todo integral, es naturalista, ya que se centra en la lógica interna de la realidad, es abierta y humanista. Este tipo de enfoque busca reivindicar lo subjetivo, lo particular y significativo para la comprensión de la realidad social (Sandoval, 1996). Asimismo, hay autores que asocian la investigación cualitativa con aquella que produce datos descriptivos (Pérez, 1994; Rodríguez, Gómez \& Gil, 1996).

El método de la presente investigación es hermenéutico por cuanto busca interpretar la realidad encontrada (García, Cantellan \& Reyes, 2018), para lo cual se realizaron entrevistas como técnica de recolección de la información, más específicamente de tipo individual estructurada, la cual "se caracteriza por la preparación anticipada de un cuestionario guía que se sigue, en la mayoría de las ocasiones de una forma estricta aun en su orden de formulación" (Sandoval, 1996, p.144). Para ello, se diseñó un instrumento con su respectivo consentimiento informado.

El análisis de resultados se realizó por medio del análisis de contenido que es una técnica de interpretación y análisis de textos de varios tipos, cuya capacidad de acoger contenido nos permite descubrir información de diversos fenómenos y aspectos de la vida social, además guarda relación con procedimientos de análisis del lenguaje (Abela, 2002, Pérez, 1994).

La población abordada fueron funcionarios que laboran y/o coordinan procesos relacionados con la atención integral en centros penitenciarios y carcelarios de Colombia, el muestreo es de tipo caso típico, debido a que este tipo de muestreo se realiza con la ayuda de informantes claves y busca dar a conocer los rasgos más comunes de una realidad. "La definición de 'típico' cualitativamente se construye a partir del consenso de opiniones entre informantes clave, buenos conocedores de la realidad bajo estudio" (Sandoval, 1996, p. 123). Este tipo de muestreo permite conocer lo que es normal o usual en un grupo. Para el presente caso se llevaron a cabo entrevistas de informantes claves de cinco de los ocho centros con Madres e infantes que tiene Colombia a cargo del INPEC (RM Popayán, RM Ibagué, RM Medellín, RM Jamundí, RM Bucaramanga). A continuación, se muestra la distribución geográfica de la muestra del estudio: 
Figura 1. Distribución geográfica de los centros penitenciarios participantes

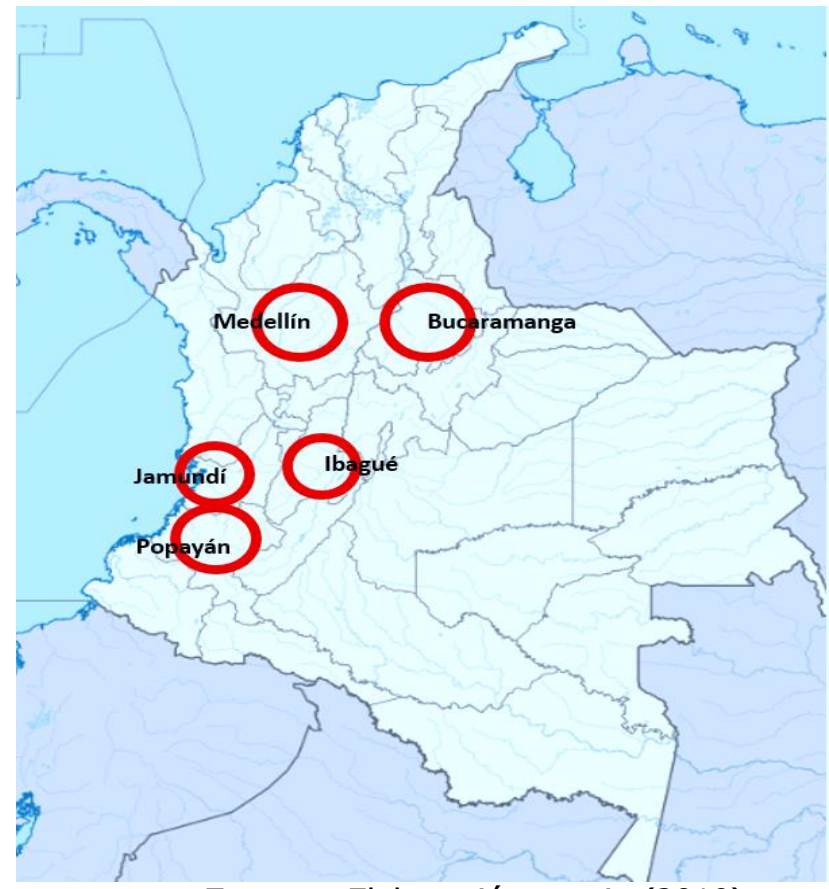

Fuente: Elaboración propia (2019).

\section{Resultados}

Se optó por un análisis de tipo inductivo, propio de la investigación cualitativa de tal forma que dio lugar a una síntesis descriptiva y abstracta de los datos (McMillan \& Schumacher, 2005).

A continuación, se presentan las tablas resumidas, resultado del análisis cualitativo realizado, en donde se encuentran los datos sociodemográficos de las profesionales entrevistadas, la caracterización de la población de madres e infancia residente en Centros penitenciarios, el análisis del programa que se ofrece desde los centros penitenciarios enfocado en la atención integral a primera infancia, y las propuestas socioeducativas de atención integral a la primera infancia:

Tabla 1.

Síntesis de datos sociodemográficos de las entrevistadas

\begin{tabular}{cc}
\hline Datos & Personal entrevistado en los Centros de reclusión \\
\hline Sexo & $\mathrm{F}(100 \%)$ \\
Nivel educativo & Profesional $(100 \%)$ \\
Estudios realizados que le permitieron el & Derecho $(20 \%)$ \\
acceso al ejercicio de las funciones & Psicología $(20 \%)$ \\
profesionales & Una licenciatura $(60 \%)$
\end{tabular}


Cargo y función profesional principal ejercida en la Institución

Tiempo trabajando en la institución
Directora de la reclusión (20\%)

Directora de Programas de Primera Infancia (20\%)

Coordinadora del hogar infantil $(20 \%)$

Agente de intervención educativa y/o social (40\%)

Elaboración propia (2019).

Entre 4 y 26 años

Nota: Centros de reclusión: RM Popayán, RM Ibagué, RM Medellín RM Jamundí RM Bucaramanga

\section{Caracterización de la población de madres e infancia residente en Centros penitenciarios:}

Tabla 2.

Número de mujeres y menores residentes actualmente en los centros de reclusión

\begin{tabular}{ccccccc}
\hline & RM & RM & RM & RM Jamundí & RM \\
Bucaramanga & Totales \\
\hline $\begin{array}{c}\text { Número de } \\
\text { mujeres } \\
\text { residentes }\end{array}$ & 5 madres & Ibagué & Medellín & & 23 \\
$\begin{array}{c}\text { actualmente } \\
\text { (gestantes) }\end{array}$ & gestantes & 5 mujeres & 6 mujeres & 4 mujeres & \\
$\begin{array}{c}\text { Número de } \\
\text { mujeres }\end{array}$ & 7 madres & 7 madres & 6 madres & 10 madres & 10 madres & 40 \\
$\begin{array}{c}\text { residentes } \\
\text { actualmente } \\
\text { Número de } \\
\text { menores }\end{array}$ & 7 niños & 7 niños & 6 niños & 10 niños & 10 niños & 40 \\
residentes & & & & & & \\
actualmente & & & & & & \\
\hline
\end{tabular}

Elaboración propia (2019).

Tabla 3.

Caracterización de la población de madres e infancia residente en Centros penitenciarios en lo relacionado a necesidades, capacidades y características

\begin{tabular}{ccc}
\hline $\begin{array}{c}\text { Categorías } \\
\text { predeterminadas }\end{array}$ & Resumen de resultados & $\begin{array}{c}\text { Temas que } \\
\text { emergen }\end{array}$ \\
\hline $\begin{array}{c}\text { Necesidades principales } \\
\text { de madres e hijas/os }\end{array}$ & $\begin{array}{c}\text { Se observaron cinco microcategorías asociadas a } \\
\text { las necesidades principales de madres e hijas/os, } \\
\text { las cuales están relacionadas con: Mejora de } \\
\text { infraestructura; Tiempo de calidad (madre e hijo); }\end{array}$ & $\begin{array}{c}\text { Infraestructur } \\
\text { Red familiar de apoyo; Necesidad de vínculos; y }\end{array}$ \\
& Atención médica. & Vinculo: \\
& Las microcategorías que aparecen con mayor & Madre e Hijos
\end{tabular}


frecuencia son: Tiempo de calidad entre madre e hijo/a y Red familiar de apoyo. Red familiar Capacidades principales de madres e hijas/os

Se observaron seis microcategorías (Pautas de

crianza; Interiorización de aprendizajes del Pautas de programa; Protectoras; Adaptabilidad al contexto; Formación de vínculos filiales y Destrezas laborales) Protección identificadas como Capacidades principales de madres e hijas/os en los centros penitenciarios. Las Adaptabilidad microcategorías que aparecen con mayor al contexto frecuencia son: Interiorización de aprendizajes del programa y madres protectoras. Vínculos

Características principales que presentan las madres y sus hijas/os
Se observaron seis microcategorías (Protección;

Resiliencia; Compromiso; Amor; Esperanza y

Participación) asociadas a las Características

principales que presentan las madres y sus hijas/os

en los centros penitenciarios. Las microcategorías

que aparecen con mayor frecuencia son:

Protección, Resiliencia y Participación.

Elaboración propia (2019).

\section{Análisis del programa que se ofrece desde los centros penitenciarios enfocado en la atención integral a primera infancia.}

Tabla 4.

Análisis del programa que se ofrece desde los centros penitenciarios enfocado en la atención integral a primera infancia

\begin{tabular}{|c|c|c|}
\hline $\begin{array}{c}\text { Categorías } \\
\text { predeterminadas }\end{array}$ & Resumen de resultados & $\begin{array}{l}\text { Temas que } \\
\text { emergen }\end{array}$ \\
\hline \multirow{4}{*}{$\begin{array}{l}\text { Descripción del } \\
\text { programa de } \\
\text { atención integral }\end{array}$} & $\begin{array}{l}\text { Se observaron ocho microcategorías (Desarrollo de } \\
\text { habilidades motrices; Desarrollo de habilidades }\end{array}$ & Desarrollo integral \\
\hline & sociales; Desarrollo integral; Desarrollo del lenguaje; & Derechos del/a \\
\hline & $\begin{array}{l}\text { Respeto por los Derechos; Desarrollo de habilidades } \\
\text { artísticas; Actividades lúdicas y pedagógicas y }\end{array}$ & niño/a \\
\hline & $\begin{array}{l}\text { Fortalecimiento de vínculos afectivos) emergentes } \\
\text { de la categoría predeterminada: Descripción del }\end{array}$ & $\begin{array}{l}\text { Actividades lúdicas } \\
\text { y pedagógicas }\end{array}$ \\
\hline
\end{tabular}


programa de atención integral.

Vínculos afectivos

Cuidados prenatales

a niños/as y madres

Infraestructuras,

espacios y recursos

del programa
Aspectos

identificados cómo

"buenas prácticas"

Características de

un día Normal

(entre semana)
Se observaron nueve microcategorías (Programa de seguimiento para el cuidado de la salud; Controles prenatales; Alimentación saludable; Sensibilización sobre fortalecimiento de vínculos afectivos; Sensibilización sobre estimulación; Sensibilización sobre importancia de la lactancia, Acompañamientos para evitar recaídas en adicciones; Sensibilización sobre cuidados prenatales y Curso bicoprofiláctico) emergentes de la categoría predeterminada:

Cuidados prenatales a niños/as y madres. Se observaron cinco microcategorías (Sesiones para ejercicios profilácticos; Área adecuada para madres e hijos/as; Sala adecuada para trabajo con familias; Recorridos a salas dentro del centro penitenciario; Condiciones de hacinamiento) emergentes de la categoría predeterminada: Infraestructuras, espacios y recursos del programa. Destacando Área adecuada para madres e hijos/as, como la microcategoría más frecuente junto con condiciones de hacinamiento o espacios reducidos.

Se observaron siete microcategorías (Aprendizaje exploratorio; Desarrollo de Habilidades de escritura; Fortalecimiento del vínculo afectivo; Desarrollo de autonomía; Pautas de crianza, Cuidados de salud; Control parental) relacionadas con la categoría predeterminada: Aspectos identificados cómo "buenas prácticas". Siendo la microcategoría Fortalecimiento del vínculo afectivo la más frecuente.

\section{Se observaron 15 microcategorías (Ingreso a} espacio infantil; Desayuno; Almuerzo; Refrigerio; Actividades de arte y literatura; Finalizan la tarde
Cuidado prenatal

Vínculos afectivos

Área adecuada para madres e hijos/as

Condiciones de hacinamiento 0 espacios reducidos

Aprendizajes y

Desarrollo de Habilidades a partir de pautas de crianza

Vínculo afectivo

Actividades rectoras 
Características de un día Normal (fin de semana) con sus madres; Duermen con sus madres; Actividades de exploración en el medio; Actividades de arte y literatura; Sensibilización sobre el medio exterior; Actividades lúdicas, Descanso; Baño; Desarrollo de habilidades motrices y Cena); relacionadas con Características de un día Normal entre semana.

Se observaron 10 microcategorías (Salen a compartir con la familia; Sensibilización a madres con películas; Permanencia de niños con sus madres en el pabellón; Espacio para alimentación; Asistencia a zona de visitas; Actividades lúdicas; Desayuno; Espacio para hacer deportes; Almuerzo; Espacio de lectura) asociadas a las Características de un día Normal en fines de semana. Por otra parte, las microcategorías más frecuentes fueron: Salen a compartir con la familia, Permanencia de niños con sus madres en el pabellón y Asistencia a zona de visitas.

Elaboración propia (2019).

\section{Propuestas socioeducativas de atención integral a la primera infancia}

Tabla 5

Propuestas socioeducativas de atención integral a la primera infancia

\begin{tabular}{lll}
\hline \multicolumn{1}{c}{$\begin{array}{c}\text { Categorías } \\
\text { predeterminadas }\end{array}$} & \multicolumn{1}{c}{ Resumen de resultados } & $\begin{array}{c}\text { Temas que } \\
\text { emergen }\end{array}$ \\
\hline Desafíos respecto & Se observaron ocho microcategorías (Formación en & Formación en valores \\
a la atención & valores, Fortalecimiento vínculo madre- hijos/as; Apoyo & \\
integral & interinstitucional para intervenciones; Desarrollo de auto- & Desarrollo de \\
& concepto del niño; Extender el plan de formación del CDI & espacios adicionales \\
& al ámbito familiar; Creación de una casa hogar anexa al & de tipo comunitario y \\
& centro penitenciario; Mejorar condiciones alimenticias & trabajo con familia \\
& para mujeres y Concientizar a madres sobre pautas de & \\
& crianza y valores) derivadas de la característica &
\end{tabular}


predeterminada Desafíos respecto a la atención integral.

La microcategoría más frecuente fue: Formación en

valores.

Medidas

Se observaron cinco microcategorías (Sensibilizar a

Desarrollo de

socioeducativas de

madres sobre pautas de crianza; Sensibilizar al personal

espacios adicionales

mejora

de custodia respecto las relaciones sociales con hijos de

de tipo comunitario y

las madres internas; Sensibilizar a profesionales sobre la

trabajo con familia

importancia e impacto de su labor; Mayor compromiso

por parte de la red de apoyo familiar; Seguimiento

Red de apoyo

formativo a niños posterior a egreso del centro

penitenciario) identificadas a partir de la microcategoría

familiar para

predeterminada: Medidas socioeducativas de mejora.

internos (madres e

hijos) y Seguimiento

a niños/as posterior

a egreso del centro

penitenciario

\section{Elaboración propia (2019).}

\section{Conclusiones y discusiones}

De acuerdo a la revisión teórica realizada y el análisis de resultados sobre la situación socioeducativa de la infancia residente en los centros penitenciarios en Colombia, se puede decir que dicha situación es variante de acuerdo al centro penitenciario que se analice, como se pudo observar en el Encuentro Académico: acción de la política: garantía de derechos de la primera infancia en centros de reclusión de mujeres (INPEC, 2017b) y en las entrevistas realizadas, sin embargo comparten varios rasgos en común.

Sin duda alguna, se opta por asumir la posición de argumentar a favor de una infancia al lado de su progenitora a pesar de las condiciones diferentes que pueda presentar el entorno ya que existen más razones de peso positivas (que sustentan la importancia del crecimiento de los/as infantes al lado de su madre) que negativas. En el caso de Colombia, estos espacios deberán tener una infraestructura que responda al adecuado desarrollo del embarazo de mujeres gestantes, sindicadas o condenadas; además debe contar con un ambiente propicio para el correcto desarrollo integral de los niños, niñas y madres lactantes residentes en estos centros (Congreso de Colombia, 2014, Ley 1709). Adicionalmente, de acuerdo a las presentes conclusiones extraídas del estudio, deberán optar por modelos de enfoque comunitario que han sido exitosos en otros países como las unidades dependientes de madres o unidades externas de Madres (Añaños, Fernández \& Llopis, 2013; Del Pozo \& Añaños, 2010, Del Pozo \& Gil, 2013). 
De este modo, se logró realizar una caracterización general de la población de madres e infancia residentes en los centros penitenciarios de Colombia teniendo como soporte los datos proporcionados por cinco (5) de los ocho (8) centros de reclusión de mujeres existentes en el país a cargo del INPEC que actualmente cuentan con niño/s y/o mujeres gestantes, en los cuales existe un total de veintitrés (23) madres gestantes y cuarenta (40) menores de tres años con sus madres. Las necesidades mayormente marcadas de esta población se encuentran referidas a la infraestructura, el vínculo afectivo: madre-hijo/a y la red familiar.

En cuanto a infraestructura, es importante recordar que la legislación nacional, establece que se deben realizar adaptaciones de tratamiento en cuanto a infraestructuras, programas, medidas, etc., en pro de la infancia y madres en periodo de gestación y lactancia en los centros penitenciarios, esto de acuerdo a la Ley 1709 del 20 de enero de 2014 (INPEC, 2014).

En cuanto al vínculo relacional madre-hijo/a y vinculo o red familia de apoyo, vale la pena mencionar que el primero se entiende como una necesidad, teniendo en cuenta que dicho vínculo afectivo generalmente es visto como factor protector sustancial de la salud mental del infante; siempre que exista dicho vinculo, la madre tratará de proveer al niño o la niña, quien inicialmente es completamente dependiente (Porras \& Lerma, 2015, Rúa, 2015) y el segundo al ser de gran importancia para el desarrollo de la socialización del menor, requiere de la apertura de espacios que fomenten el fortalecimiento de los vínculos con la familia extensa y sus hijas/os (Defensoría del Pueblo, 2010; Jiménez, 2008).

Asimismo, entre las capacidades y características principales de estas madres con sus hijos, se resaltan: pautas de crianza que tienen para con sus hijos, la protección que brindan, la adaptabilidad al contexto que poseen, los vínculos que llegan a formar, y la resiliencia, todas, particularidades propias de madres que, en este caso, presentan una condición de encierro.

En relación con el análisis del programa que se ofrece desde los centros penitenciarios enfocado en la atención integral a primera infancia se pueden resaltar aspectos con gran prevalencia como el desarrollo integral, expuesto como uno de los aspectos fundamentales de los programas. Con respecto a esto, el ser humano durante su proceso de desarrollo establece una triple interacción: con sí mismo, con los otros y con su ambiente (Amar, 2011; Amar, Abello \& Tirado, 2004), siendo la fusión de estas tres lo que constituye el desarrollo integral en todas sus dimensiones: cognitiva, comunicativa, socioafectiva, física, ética, estética y trascendental. Asimismo, dentro de estos programas resaltan los derechos del/a niño/a, las actividades lúdicas y pedagógicas, la importancia conferida a los vínculos afectivos y la interacción con los pares, factores que inciden enormemente en el aprendizaje y el desarrollo (Castro \& Morales, 2015; Papalia, Duskin y Martorell, 2012).

Con respecto a los cuidados prenatales, se resaltan nuevamente los vínculos afectivos y 
todos los cuidados ofrecidos que según Jewell, Triunfo, y Aguirre (2004) impactan positivamente la salud del/la niño/a. En este sentido la ley 1709 (Congreso de Colombia, 2014) responsabiliza de varios aspectos a los centros penitenciarios y al Instituto Colombiano de Bienestar Familia (ICBF), relacionados con el tratamiento a infancia, sin embargo, el cuidado prenatal (especialmente en el ámbito de la salud) termina siendo más responsabilidad del centro que del ICBF. De acuerdo a las entrevistas realizadas, se puede confirmar la importancia de los cuidados prenatales que en este caso denotan en casi todos los casos situaciones positivas y de autonomía, entre otras cosas por la posibilidad que tienen las madres de salir del centro penitenciario a los siete meses de gestación para la preparación de su parto.

Para el caso de infraestructuras, espacios y recursos del programa de atención, se resaltan dos puntos contrastantes: por un lado, se encuentra la percepción de la existencia áreas adecuadas para madres e hijos/as y por otro lado se relatan condiciones de hacinamiento o espacios reducidos. Referente a esto, es conocido que las condiciones de vida que se presentan en una prisión en muchos casos son inhumanas y no alcanzan a suplir las necesidades básicas como es el caso de algunas cárceles colombianas (Mahecha \& Gutiérrez, 2015). Y aunque en el presente estudio no se despliegan situaciones inhumanas, de acuerdo a lo expuesto en las entrevistas, si se presentan condiciones de hacinamiento o espacios reducidos. En Colombia, la norma concreta que los centros penitenciarios deberán contar con una infraestructura que garantice a las mujeres gestantes un adecuado desarrollo del embarazo, debe ofrecerse un contexto de convivencia que no impactante negativamente en los menores. El ICBF en coordinación con la Unidad de Servicios Penitenciarios y Carcelarios (USPEC) establecen las condiciones que deben cumplir los establecimientos de reclusión de mujeres con el fin de resguardar los derechos de los niños y las niñas que conviven con sus madres (Congreso de Colombia, 2014, Ley 1709).

En cuanto a buenas practicas, se denota el fomento de aprendizajes y desarrollo de habilidades a partir de pautas de crianza y el favorecimiento de vínculos afectivos fuertes entre madres e hijos/as, que adicionalmente son visibles en la descripción de los días normales (entre semana y fin de semana) en donde se hace alusión a las actividades rectoras: el arte (Ministerio de Educación Nacional, 2014a), el juego (MEN, 2014b), la literatura (MEN, 2014c), la exploración del medio (MEN, 2014d). En estos relatos sobre días normales, también se resaltan los espacios en familia, específicamente en los fines de semana.

En cuanto al planteamiento medidas socioeducativas de atención integral, en los desafíos descritos por las entrevistadas, se enfatiza, por un lado, en la formación en valores y por el otro, en el desarrollo de espacios adicionales de tipo comunitario y trabajo con familia. La formación en valores desde siempre ha sido una preocupación de la sociedad, especialmente en el ámbito penitenciario; en los años 30 bajo la Dictadura Franquista las comunidades religiosas se encargaban de atención con la finalidad de "intensificar los valores morales" dentro de los establecimientos penitenciarios, asumiendo funciones no establecidas en las 
cárceles masculinas, dominando distintas facetas en la vida de las mujeres, convirtiendo los centros de reclusión en "prisiones-conventos" (Yagüe, 2007) por lo que se sugieren programas y unidades educativas que incorporen formación en valores y habilidades para la convivencia (Del Pozo, Jiménez \& Turbi, 2013; Enjuanes, García \& Longoria, 2014).

En cuanto al desarrollo de espacios adicionales de tipo comunitario y trabajo con familia, no solo se plantea como un desafío, sino que, además, desde la percepción de las entrevistadas, es mencionado como una de las medidas socioeducativas de mejora, dado que este tipo de espacios constituye una de las modalidades más sobresalientes de atención y educación para infancia residente en centros penitenciarios, denominado modelo alternativo de cumplimiento "unidad externa" en entorno comunitario, que es de régimen abierto y externo (Del Pozo, 2017a). Según lo extraído de las entrevistas, los espacios comunitarios, se convierten en una medida de mejora para la atención integral de madres e hijos recluidos en centros penitenciarios. Estas iniciativas están presentes en países como Inglaterra, Polonia, Finlandia, Alemania, Holanda, Australia y Estados Unidos, entre otros y alejan realmente a mujeres embarazadas o madres y pequeños de las prisiones, ofreciéndoles la oportunidad de cumplir sus sentencias dentro de una comunidad y entornos más favorables. (Cortázar, et al, 2015), permitiendo que los niños y niñas no sean concebidos como internos no penados en prisión (Gea, 2017) y procurando por un ambiente familiar y comunitario que debe estar dirigido al desarrollo infantil temprano (Raczynski, 2006).

El entorno comunitario cobra relevancia debido a que el infante ingresará a los tres años a ese medio en su totalidad, de ahí que se planee como otra medida socioeducativa de mejora el fortalecimiento de la red de apoyo familiar para internos (madres e hijos) y seguimiento a niños/as posterior al egreso del centro penitenciario, ya que el trabajo construido en centro de reclusión pierde continuidad, por ello es importante la disposición de los socio-entornos positivos que supongan el desarrollo adecuado de los menores internos, y la apertura de espacios que fomenten el fortalecimiento de los vínculos con la familia extensa y sus hijas/os del exterior (Jiménez, 2008) de este modo, cuando egresen del entorno penitenciario, la familia podría darle continuidad al proceso de una manera más segura. Para todo esto y teniendo en cuenta que la mayoría de problemas sociales tienen razón y causa en la educación, es importante resaltar el papel de la pedagogía y la educación social en este estudio ya que son vías alternativas a las prácticas educativas convencionales que con frecuencia son poco coherentes con las circunstancias cotidianas de la vida de los/las reclusos/as (Caride \& Gradaílle, 2013, Del Pozo, 2017b; Del pozo \& Ramírez, 2017).

Para finalizar, se puede decir que la situación socioeducativa vinculada a la primera infancia residente en los centros penitenciarios y carcelarios de Colombia, es variada pero estable, ya que ha mostrado tener, en la mayoría de los casos, condiciones relativamente adecuadas para la atención a primera infancia y sus madres, sin embargo es importante puntualizar en la posibilidad de entornos socio-comunitarios como una de las principales conclusiones y recomendaciones, teniendo en cuenta que la ley hace alusión a procurar por 
el correcto desarrollo psicosocial de los niños y niñas menores de tres años residentes en centros penitenciarios (Congreso de Colombia, 2014, Ley 1709) y para esto, debe ofrecerse un contexto de convivencia sin efectos negativos. La forma de garantizar que no exista impacto alguno en esta población, es generando un entorno con las mismas condiciones externas, que si bien, en muchos casos no son las mejores (niños/as que viven en pobreza extrema, situaciones de violencia doméstica, entre otros), no es razón o excusa para dejar de plantear alternativas más adecuadas.

\section{Referencias}

Abela, J. (2002). Las técnicas de análisis de contenido: una revisión actualizada. Recuperado de http://mastor.cl/blog/wp-content/uploads/2018/02/Andreu.-analisis-decontenido.-34-pags-pdf.pdf

Alcocer, L. (2011). Análisis de la tríada familia-escuela-sociedad: un estudio comparativo. Revista Educación y Ciencia, 4(12), 51-61. Recuperado de http://educacionyciencia.org/index.php/educacionyciencia/article/view/104

Almeda, E., Di Nella, D. \& Navarro, C. (2012). Mujeres, cárceles y drogas: datos y reflexiones (Women, Prisons and Drugs: Data and Reflections). Oñati Socio-legal series, 2(6) 122- 145.

Almeda, E. \& Di Nella, D. (2017). Mujeres y cárceles en América Latina: perspectivas críticas y feministas. Papers: revista de sociologia, 102(2), 183-214.

Amar, J., Abello, R. \&Tirado, D. (2004). Desarrollo infantil y construcción de mundo social. Barranquilla, Colombia: Ediciones Uninorte

Amar, J. (2011). Educación infantil y desarrollo social. Investigación y Desarrollo, (7) 1-17.

Añaños, F. (2012). Las mujeres en las prisiones. La Educación Social en contextos de riesgo y conflicto. Pedagogía Social. Revista Interuniversitaria, (19), 169-170. Recuperado de http://www.redalyc.org/pdf/1350/135025474012.pdf

Añaños, F., Fernández, M. \& Llopis, J. (2013) Aproximación a los contextos en prisión una perspectiva socioeducativa. Pedagogía Social, Revista Interuniversitaria, (22), 13-28. Doi: 10.7179/PSRI_2013.22.02

Ayuso, A. (2000). La intervención socioeducativa en el tratamiento penitenciario. Pedagogía social: Revista interuniversitaria, (6), 73-99.

Borja, M., Jiménez, M., Narváez, V. \& Polo, G. (2017), Infancia y vulneración de derechos: Aproximación desde el ámbito penitenciario. En F, Del Pozo. Exclusión mujeres y prisión en Colombia un caso en la región caribe (pp. 111 - 132). Barranquilla Colombia: Editorial Universidad del Norte

Campo, L. (2014). El desarrollo del autoconcepto en niños y niñas y su relación con la interacción social en la infancia. Psicogente, 17(31), 67-79. Doi: 


\subsection{1/psico.17.31.422}

Caride, J. \& Gradaílle, R. (2013). Educar en las cárceles: nuevos desafíos para la educación social en las instituciones penitenciarias. Revista de educación, (360), 36-47. Doi: 10.4438/1988-592X-RE-2013-360-219

Castro, M. \& Morales, M. (2015). Los ambientes de aula que promueven el aprendizaje, desde la perspectiva de los niños y niñas escolares. Revista Electrónica Educare, 19(3), 1-32. Doi: http://dx.doi.org/10.15359/ree.19-3.11

Cecchini, S., Filgueira, F. \& Robles, C. (2014). Sistemas de protección social en América Latina y el Caribe. Una perspectiva comparada. Santiago de Chile: Naciones Unidas/CEPAL.

Congreso de Colombia (2014). Ley 1709. Artículo 153. Recuperado de http://www.defensoria.gov.co/public/Normograma\%202013_html/Normas/Ley_170 9_2014.pdf

Constitución política de Colombia [Const.] (1991). Artículo 44. Recuperado de 2017 de http://www.constitucioncolombia.com/titulo-2/capitulo-2/articulo-44

Cortázar, A., Fernández, P., Léniz, I., Quesille, A., Villalobos, C., \& Vielma, C. (2015). ¿Qué pasa con los hijos de madres encarceladas? Cómo amortiguar los efectos nocivos para los niños cuyos padres están privados de libertad. Santiago, Chile: Instituto de Políticas Públicas, Universidad Diego Portales.

Defensoría del Pueblo. (2010). El derecho de los niños, niñas y adolescentes a vivir en una familia: la situación de los Centros de Atención Residencial estatales desde la mirada de la Defensoría del Pueblo. Recuperado de http://repositorio. minedu.gob.pe/handle/123456789/603

Del Pozo, J. \& Añaños, I. (2010). Experiencias y Programas Socioeducativos en el Medio Penitenciario cerrado y abierto con Mujeres e Infancia. En F.T. Añaños (coord.), Las mujeres en las prisiones. Educación social en contextos de riesgo y de conflicto (Pp. 235-260). Barcelona: Gedisa

Del Pozo, F. (2012). Educación social para la salud: proyección, acción y profesionalización. Revista Médica de Risaralda, 19(1), 75-80. Doi: http://dx.doi.org/10.22517/25395203.7881

Del Pozo, F., Jiménez, F. \& Turbi, Á. (2013). El tratamiento con mujeres: Actuación socioeducativa y sociolaboral en prisiones. Pedagogía Social. Revista Interuniversitaria, (22), 57-72. Doi: 10.7179/PSRI_2013.22.05

Del Pozo, F. \& Gil, F. (2013) conocimiento pedagógico y crisis social. Los efectos de la crisis en las prisiones. En S, Torio. O, García. Peña, J. C, Fernández (coord.) Crisis social y el estado del bienestar: las respuestas de la pedagogía social, (pp. 65-71) Oviedo, España: Academia. Recuperado de: https://dialnet.unirioja.es/servlet/articulo?codigo $=4481460$ 
Del Pozo, F. \& Idárraga, M. (2015). Retos del tratamiento penitenciario en Colombia: enfoque y acción diferencial de género desde la perspectiva internacional. Revista Criminalidad, 57(1), 9-25. Recuperado de: http://www.scielo.org.co/pdf/crim/v57n1/v57n1a02.pdf

Del pozo, F. (2017a). El Enfoque Diferencial de Género en el Ámbito Penitenciario: Marco Nacional e Internacional de la Atención de la Primera Infancia, en INPEC GOBIERNO DE COLOMBIA - ESTRATEGÍA DE CERO A SIEMPRE (Organizadores). Encuentro académico: acción de la política: garantía de derechos de la primera infancia en centros de reclusión de mujeres. Conferencia llevada a cabo en congreso académico, Bogotá Colombia.

Del pozo, F. (2017b). Exclusión mujeres y prisión en Colombia En Colombia: Un caso en la Región Caribe. Colombia: Editorial Universidad del Norte.

Del pozo, F. \& Ramírez, M. (2017). Women Prisoners in the Colombian Caribbean and Socialeducative Action: Multicultural Faces in the Feminization of Poverty, Conflict and Social Exclusion. Procedia-Social and Behavioral Sciences, (237), 216-222. Doi: 10.1016/j.sbspro.2017.02.066

Enjuanes, J., García, F. \& Longoria, B. (2014) La Unidad Terapéutica y Educativa del Centro Penitenciario de Villabona, un nuevo modelo penal de reinserción social. Educación Social. Revista de Intervención Socioeducativa, (57), 33-47. Recuperado de https://www.siis.net/documentos/ficha/217376.pdf

García, M. Cantellan, M. \& Reyes, A. (2018). Un acercamiento a la investigación cualitativa en la disciplina del diseño. RIDE Revista Iberoamericana para la Investigación y el Desarrollo Educativo, 8(16), 535-556. Doi: http://dx.doi.org/10.23913/ride.v8i16.357

Gea, M. (2017). Maternidad en prisión. Situación de los hijos e hijas que acompañan a sus madres compartiendo condena. Revista de Sociología. 102(2)9 Doi: http://dx.doi.org/10.5565/rev/papers.2339

Gobierno de Colombia. (2013). Estrategia de atención integral a la primera infancia fundamentos técnicos, políticos y de gestión. Recuperado de http://www.deceroasiempre.gov.co/QuienesSomos/Paginas/QuienesSomos.aspx

González, J. (2001). El paradigma interpretativo en la investigación social y educativa: nuevas respuestas para viejos interrogantes. Cuestiones pedagógicas, (15), 227246. https://idus.us.es/xmlui/bitstream/handle/11441/12862/file_1.pdf?sequence

INPEC-Instituto Nacional Penitenciario y Carcelario. (2014). Informe estadístico. Julio de 2014. 
INPEC-Instituto Nacional Penitenciario y Carcelario. (2017a). Informe estadístico \# 7. Julio de $2017 . \quad$ Recuperado de http://www.inpec.gov.co/documents/20143/362591/07+INFORME+JULIO+2017.p df/57089e36-7d9f-67a5-65b0-087877192cb1?version=1.0

INPEC-Instituto Nacional Penitenciario y Carcelario. (2017b) Encuentro académico: acción de la política: garantía de derechos de la primera infancia en centros de reclusión de mujeres. $\quad$ Recuperado http://www.deceroasiempre.gov.co/Prensa/Paginas/e_Encuentro-AcademicoAccion-Politica-Garantia-Derechos-de-la-Primera-Infancia-en-Centros-ReclusionMujere.aspx

Jaffé, P., Pons, F. \& Rey, H. (1997). Children Imprisioned with Their Mothers: Psychological Implications. En S, Redondo. V, Garrido. J, Pérez. \& R, Barberet (Eds.). Advances in Psychology and Law (pp. 339-407): International Contributions. Berlin: Walter de Gruyter.

Jaramillo, L. (2007) Concepciones de Infancia. Zona Próxima, (8), 108-123. Recuperado de https://search.proquest.com/docview/1435673852?pq-origsite=gscholar

Jewell, R., Triunfo, P. \& Aguirre, R. (2004). Impacto de los cuidados prenatales en el peso al nacer: el caso del Uruguay. Recuperado de https://www.colibri.udelar.edu.uy/jspui/bitstream/20.500.12008/1998/1/DT\%20E\%2 02004-07.pdf

Jiménez, J. (2008). Niños, niñas y madres en prisión. Contexto y desarrollo en los centros penitenciarios españoles. Educación social en el ámbito penitenciario: mujeres, infancia y familia. (pp.155-170). Educación social en el ámbito penitenciario: mujeres, infancia y familia. Granada: Natívola.

Jiménez, M. (2012). Las mujeres en las prisiones. La Educación Social en contextos de riesgo y conflicto de Fanny T. Espacios Públicos, 15(35) 169-170. Recuperado de http://www.redalyc.org/pdf/1350/135025474012.pdf

López, N. (2009). Comunicación materno-filial en el embarazo. Cuadernos de Bioética, septiembre-diciembre, 20 (70), 303-315. Recuperado de https://dialnet.unirioja.es/servlet/articulo?codigo=3058968

López, M. (2016). Contacto precoz piel a piel madre recién nacido: beneficios fisiológicos y psicológicos. In Crescendo Ciencias De La Salud, 3(2), 189-198. Doi: http://dx.doi.org/10.21895/in cres CS.v3i2.1435

Mahecha, J. \& Gutiérrez, J. (2015). El hacinamiento en las cárceles de Colombia: análisis de tres consecuencias e impactos que genera en los reclusos. Nueva Granada, España: Universidad Militar.

Marcó, F. (2014). Calidad del cuidado y la educación para la primera infancia en América Latina: Igualdad para hoy y mañana. Santiago de Chile: CEPAL. 
McMillan, J. \& Schumacher, S. (2005). Investigación educativa una introducción conceptual. España: Pearson educación.

MEN (Ministerio de Educación Nacional). (2014a) Documento 21: El arte en la Educación Inicial.

Recuperado

de http://www.deceroasiempre.gov.co/Prensa/CDocumentacionDocs/Documento-N21Arte-educacion-inicial.pdf

MEN (Ministerio de Educación Nacional). (2014b) Documento 22: El juego en la Educación Inicial. https://www.educacionbogota.edu.co/archivos/Temas\%20estrategicos/Educacion_i nicial/2016/Juego_EducacionInicial.pdf

MEN (Ministerio de Educación Nacional). (2014c) Documento 23: La literatura en la Educación Inicial. Recuperado de http://www.deceroasiempre.gov.co/Prensa/CDocumentacionDocs/Documento-N23literatura-educacion-inicial.pdf

MEN (Ministerio de Educación Nacional). (2014d). Documento 24: La exploración del medio en la Educación Inicial. Recuperado de http://www.educacionbogota.edu.co/archivos/Temas\%20estrategicos/Educacion_i nicial/2016/ExploracionMedio_EducacionInicial.pdf

Mir, M., Batle, M \& Hernández, M. (2009). Contextos de colaboración familia-escuela durante la primera infancia. Investigación Innovación Educativa y Socioeducativa, 1(1), 4568.

Moneta, M. (2014). Apego y pérdida: redescubriendo a John bowlby. Revista chilena de pediatría, 265-268. Doi: http://dx.doi.org/10.4067/S0370-41062014000300001

Morillo, J. \& Montero, L. (2010). Lactancia materna y relación materno filial en madres adolescentes. Enfermería Global, (19). Recuperado de: http://scielo.isciii.es/scielo.php?pid=S169561412010000200019\&script=sci_arttext \&tlng=en

Naredo, M. (2007). Reclusas con hijos/as en la cárcel. En Elizabeth Alameda y Encarna Boledón. En E, Boledón. Y E, Almeda. (Ed). Mujeres y Castigo: Un Enfoque SócioJurídico y De Género (pp: 263-275). Barcelona: Dykinson.

Papalia, D., Duskin, R. \& Martoell, G. (2012). Desarrollo Humano. Malaga, España: Mc Graw Hill

Parlamento Europeo. (2008). Informe sobre la situación especial de las mujeres en los centros penitenciarios y las repercusiones de la encarcelación de los padres sobre la vida social y familiar. Bruselas: Parlamento Europeo.

Pérez, G. (1994). Investigación cualitativa. Retos e interrogantes I y II. Madrid: La Muralla.

Poehlmann, J. (2005). Representations of attachment relationships in children of incarcerated mothers. Child Development, (76) 679-696. Doi: 10.1111/j.1467- 


\subsubsection{1.x}

Porras, I. \& Lerma I. (2015). Construcción narrativa de la coevolución del vínculo madrehijo e institución penitenciaria. (Tesis de maestría). Universidad de Santo Tomas, Bogotá, Colombia.

Raczynski, D. (2006). Política de infancia temprana en Chile: Condicionantes del desarrollo de los niños. Santiago: Unicef. Recuperado de http://expansiva.cl/media/en_foco/documentos/19062006104331.pdf

Rico, M. \& Trucco, D. (2013). Adolescentes, Derecho a la Educación y al Bienestar Futuro. Santiago de Chile: CEPAL/UNICEF.

Rodríguez, G., Gómez, J. \& Gil, J. (1996). Métodos de la investigación cualitativa. Málaga, España: Ediciones ALJIBE.

Rúa, A. (2015). Los cuidados maternos y su relevancia en la salud mental: efectos de la primera experiencia vinculación del sujeto. Revista Electrónica Psiconex, 11(7), 113. Recuperado http://aprendeenlinea.udea.edu.co/revistas/index.php/Psyconex/article/view/24849

Sánchez, J. (2013). Paradigmas de investigación educativa: de las leyes subyacentes a la modernidad reflexiva. Entelequia: revista interdisciplinar, (16), 91-102. Recuperado de https://revistaentelequia.wordpress.com/2013/10/12/1320/

Sandoval, C. (1996). Investigación cualitativa. Colombia: Icfes.

Torres, J. (2018). La práctica de valores en el entorno de aprendizaje y desarrollo de una guía para su fortalecimiento en niños y niñas de nivel inicial II del Jardín "Lucinda Toledo" (Tesis de pregrado). Universidad central del ecuador, Quito. Recuperado de http://200.12.169.19/handle/25000/16105

UNICEF. (2005). La infancia amenazada. Recuperado de https://www.unicef.org/spanish/sowc05/childhooddefined.html

UNIFEM. (2007). Mujeres y prisión en Colombia: análisis desde una perspectiva de derechos humanos y género. Bogotá. Recuperado de http://bdigital.unal.edu.co/54279/1/9789588295282.pdf

Vila, I. (2000). Aproximación a la educación infantil: características e implicaciones educativas. Revista Iberoamericana de educación, (22), 41-60.

Yagüe, C. (2007). Madres en prisión: Historia de las cárceles de mujeres a través de su vertiente maternal. Malaga, España: COMARES. 


\section{Agradecimientos}

Agradecemos a todas las personas y entidades que con su dedicación apoyo, y tiempo hicieron posible la realización de esta investigación.

A las instituciones y personas que con su participación permitieron aportar información, conocimiento y experiencia para darle sentido a cada uno de los objetivos que se buscaron a lo largo del estudio; cabe mencionar a cada uno de los centros penitenciarios que abrieron las puertas para conocer su realidad interior, al INPEC, Estrategia de cero a siempre y Gobierno de Colombia, por la realización del Encuentro académico: acción de la política: garantía de derechos de la primera infancia en centros de reclusión de mujeres (2017), que fue de gran ayuda para conocer datos actuales y concretar relaciones con los centros penitenciarios.

A Colciencias y al programa de Jóvenes investigadores e Innovadores (con afiliación Universidad del Norte) por la oportunidad por medio de la convocatoria: 761, con la que fomentan la vocación científica en jóvenes a través de la realización de becas-pasantía en alianza con grupos de investigación y con la que se pudo llevar a cabo el presente estudio con su financiación.

A nuestros padres que siempre nos han acompañado y apoyado; a amigos/as, compañeros/as y pareja, quienes siempre han brindado su apoyo incondicional.

Viviana Ahumada Carriazo. 